\title{
Optical Levitation of Nanodiamonds by Doughnut Beams in Vacuum
}

\author{
Lei-Ming Zhou, ${ }^{1}$ Ke-Wen Xiao, ${ }^{1}$ Jun Chen, ${ }^{2}$ and Nan Zhao ${ }^{1, *}$ \\ ${ }^{1}$ Beijing Computational Science Research Center, Beijing 100193, China \\ ${ }^{2}$ Institute of Theoretical Physics and Collaborative Innovation Center of Extreme Optics, Shanxi University, Shanxi, China
}

(Dated: August 6, 2018)

\begin{abstract}
Optically levitated nanodiamonds with nitrogen-vacancy centers promise a high-quality hybrid spinoptomechanical system. However, the trapped nanodiamond absorbs energy form laser beams and causes thermal damage in vacuum. It is proposed here to solve the problem by trapping a composite particle (a nanodiamond core coated with a less absorptive silica shell) at the center of strongly focused doughnut-shaped laser beams. Systematical study on the trapping stability, heat absorption, and oscillation frequency concludes that the azimuthally polarized Gaussian beam and the linearly polarized Laguerre-Gaussian beam $\mathrm{LG}_{03}$ are the optimal choices. With our proposal, particles with strong absorption coefficients can be trapped without obvious heating and, thus, the spin-optomechanical system based on levitated nanodiamonds are made possible in high vacuum with the present experimental techniques.
\end{abstract}

Introduction-. By trapping, detecting and manipulating nano- and micro-particles [1], optical tweezers are widely used in biophysics [2-4], colloidal sciences [5], chemistry, microfluidic dynamics [6], and fundamental physics [7-15]. Because of the wide applicability and high tunablity of the optically levitated systems, several schemes [16] were proposed to realize the ground-state cooling [17], to search for non-Newtonian gravity [18] and to detect gravitational wave [19]. Particularly, it brings about more interesting phenomena and novel applications $[20,21]$ when the trapped particles have internal degrees of freedom (such as spins or electric dipoles) and enter the quantum regime.

Optically levitated nanodiamonds with nitrogen-vacancy (NV) centers [22-26] are one of the most promising candidates for implementing a spin-optomechanical hybrid system. In principle, this system can have both long spin coherence time and high quality factor of mechanical oscillation in vacuum. The electron spins of NV centers were shown to have long spin coherence time (in the order of $10^{2} \mu \mathrm{s}$ ) even in nanodiamonds of diameter about $20 \mathrm{~nm}$ [27]. When trapped in high-vacuum, the dielectric particles are predicted to have ultra-high quality factor $Q$ larger than $10^{10}[16,18,28]$. Researchers have trapped diamond particles and observed the signal from NV centers in liquid [29, 30], in air [31] and very recently in vacuum with pressure down to $\sim \mathrm{kPa}[24,25]$ and $\sim 100 \mathrm{~Pa}$ [26].

Realizing high quality mechanical oscillation requires trapping the particles in high vacuum (e.g, $10^{-6} \mathrm{~Pa}$ ) to get $Q \sim 10^{10}$. However, the high-vacuum condition usually causes the thermal damage problem, and experimentally trapping a nanodiamond in high vacuum is still very challenging. Nanodiamonds will absorb energy from the trapping laser beams due to the intrinsic defects [26] and the inevitable imperfections or graphitization [32] on diamond surface. The absorbed energy can hardly be dissipated in a high-vacuum environment, and the nanodiamonds will be quickly heated up significantly [24-26], which is unfavorable to the defect centers, or even burns out the diamond particles. Improving

\footnotetext{
*nzhao@csrc.ac.cn
}

the purity of trapped nanodiamonds is one way to reduce the heat absorption [26]. Here, we study an alternative way by engineering the trapping beams, which is in principle applicable to much wider range of particles.

It is proposed here to solve the thermal damage problem by trapping a silica-coated nanodiamond with doughnut beams [e.g., the Laguerre-Gaussian (LG) beams, see Fig. 1]. Our proposal is based on the following two observations. Firstly, recent experiments [9] show that micro-sphere made of silica can be trapped in high-vacuum without strong heat absorption because of the low absorption coefficient. Secondly, it is well-known that the cross-section intensity distribution of the doughnut beam has a dark region at the beam center. Nanodiamonds can be coated with a silica shell [24, 33], forming a core-shell structure. When the dark region of the beam coincides with the diamond core, the heat absorption will be significantly suppressed. Trapping and manipulating particles

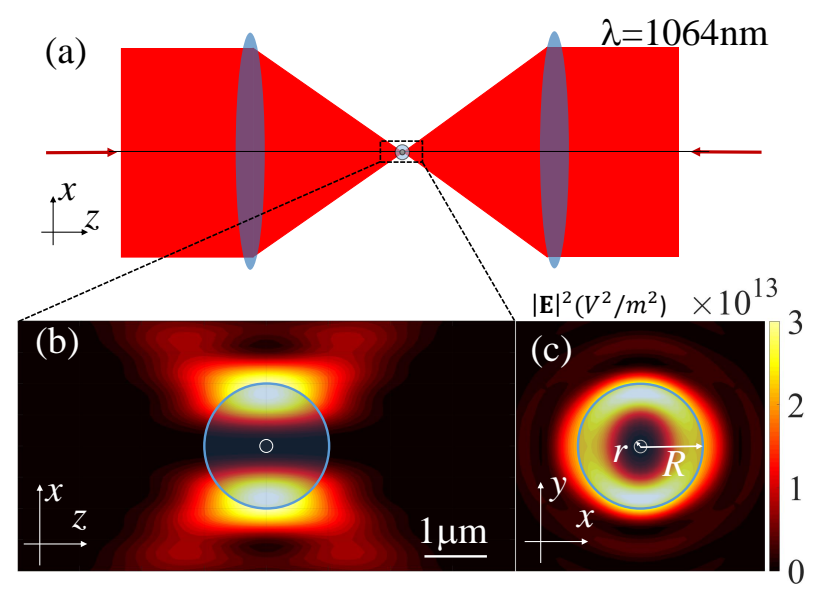

FIG. 1. (a) The schematic illustration of the system. A nanodiamond coated with a silica shell is levitated in an optical trap formed by two incoherent strongly-focused counter-propagating beams. (b) (c) Front view and side view of the intensity distribution of the two focused linearly polarized $\mathrm{LG}_{03}$ incident beams in the focal region. The circles indicate the composite particle with the core radius $r=100 \mathrm{~nm}$ and the shell radius $R=1 \mu \mathrm{m}$. Two incident beams are with total power $100 \mathrm{~mW}$. 

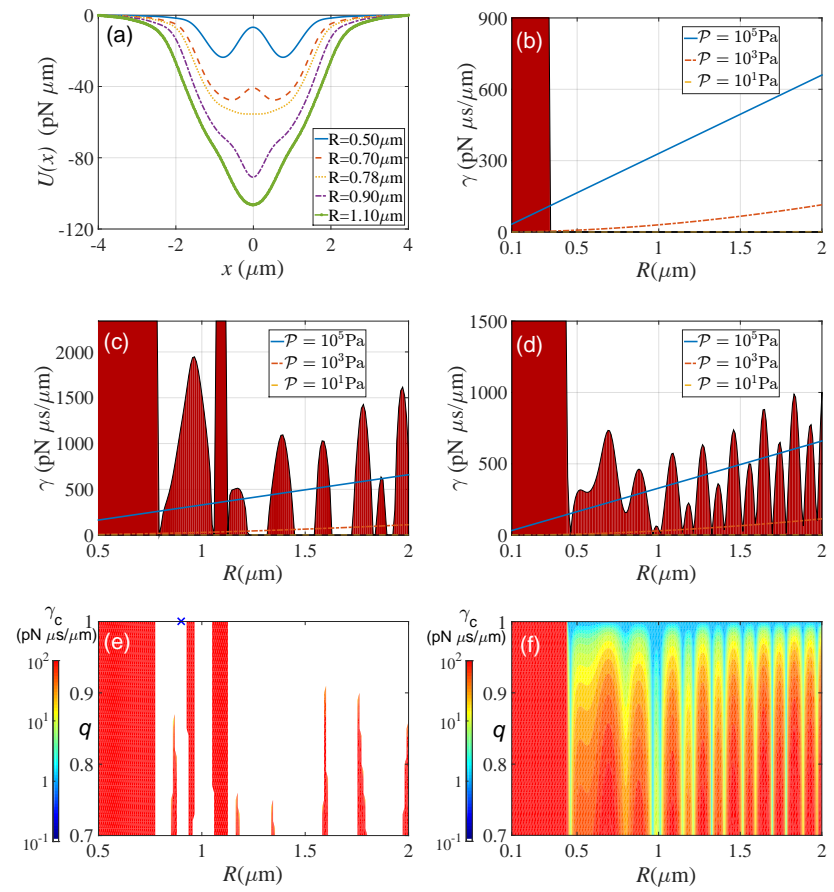

FIG. 2. (a) The transverse optical potential $U(x)$ for a coreshell spherical particle in a single linearly polarized $\mathrm{LG}_{03}$ beam with power $P=100 \mathrm{~mW}$. (b)-(d) The transverse stability diagram of a core-shell particle trapped in an azimuthally polarized beam, a linearly polarized $\mathrm{LG}_{03}$ beam and a right circularly polarized $\mathrm{LG}$ beam, in turn. The white regions are stable. The Stokes friction coefficients $\gamma$ under different pressure $\mathcal{P}$ are also plotted with lines for reference. (e) The minimum friction coefficients $\gamma_{c}$ required to stabilize the optical trap formed by the dual-beam optical tweezers with the $\mathrm{LG}_{03}$ beams, as functions of the power mismatch ratio $q$ and particle size $R$. The blue ' $X$ ' denotes a stably trapped sphere for light absorption suppression and equilibrium temperature simulation later. (f) The same as (e), but for the right circularly polarized LG beams. Other parameters used here are $\lambda=1064 \mathrm{~nm}, \mathrm{NA}=0.95$ and each beam with power $P=100 \mathrm{~mW}$ for all figures.

with doughnut beams have been investigated [34-39] in liquids. Here, we focus on the problem in vacuum which has low friction and low dissipation.

The key problem is under what condition that the composite particle can be stably trapped in vacuum with the core lying in the dark region. We numerically solve the electromagnetic wave scattering problem and show that the gradient force from a doughnut beam can form a single-well potential, as long as the particle size exceeds a critical radius $R_{\text {trans }}$ [see Fig. 2(a)]. Furthermore, the doughnut beam usually carries non-zero orbital angular momentum (OAM) and has different polarizations. The OAM and polarization affect the trapping stability and heat absorption of the particle, particularly in the case of the strongly focused beam. We systematically investigate trapping effect of doughnut beams with various OAM (e.g., the $\mathrm{LG}_{0 l}$ beams) and polarization (e.g., linearly or circularly polarized LG beams and the cylindrical vector beams [40]). By comparing the trapping stability, the heat absorption, and the oscillation frequency of the composite particle trapped in different types of doughnut beams, we conclude that the azimuthally polarized Gaussian beam and the linearly polarized $\mathrm{LG}_{03}$ beam are the optimal choices for implementing the hybrid spin-optomechanical system in vacuum.

Trapping stability-. We consider a dual-beam optical tweezers system as shown in Fig. 1(a). Two incoherent counter-propagating laser beams of wavelength $\lambda$ are focused by two identical lenses with numerical aperture NA. When the two beams with same parameters except the directions are well-aligned, the $z$ direction scattering forces from the two beams cancel each other, and the gradient forces form an optical trap near the focal point in three dimensional space.

We start the discussion from considering the strongly focused $\mathrm{LG}_{0 l}$ beams $(l>0)$. The focused beams violate the paraxial condition, and we perform numerical calculations of the focal field, following the theory developed by Richards and Wolf [41, 42]. Figures 1(b) and 1(c) show the light intensity distribution in the focal region. Similar to the paraxial case, there is a dark region along the beam propagating axis (the $z$ axis). However, in contrast to the paraxial beams whose OAM are usually well-defined and separated with the polarization degree of freedom, the orbital and polarization degrees of freedom are highly mixed around the focal point. More importantly, we will show that the trapping stability and the heat absorption are sensitive to the choice of the OAM and the polarization of the focused beams.

The focused beams provide an optical trap to the particle. As an example, Fig. 2(a) shows the trapping potential $U(x)=-\int_{-\infty}^{x} F_{x}\left(x^{\prime}\right) d x^{\prime}$ along the $x$ direction for incident $\mathrm{LG}_{03}$ beams, where $F_{x}$ is the $x$ component of the optical force $\mathbf{F}$ when the particle is displaced along the $x$ axis [43]. Generally speaking, small particles tend to be trapped at the position of maximal intensity. For incident LG beams, a small particle (e.g., with radius $R \ll \lambda$ ) will be confined in the region of the bright ring, corresponding to a double-well potential. With increasing particle size, the trapping potential is gradually changed to a single-well. The transition radius $R_{\text {trans }}$ from double-well to single-well is comparable to the radius of the bright ring. A composite particle with radius $R>R_{\text {trans }}$ will be trapped around the equilibrium position with its core locates in the dark region of the focused LG beams.

The LG beams carry OAM, which accelerates the particle in the azimuthal direction and strongly affects the trapping stability [42]. The trapping stability is determined by the force constant matrix $\mathbb{K}=\nabla \mathbf{F}$ of the focused beam at the equilibrium position (when $R>R_{\text {trans }}$ ). Because of the OAM of the LG beams, the $y$-component of the radiation force $F_{y}$ is nonzero, when the particle displacement is along the $x$ direction. In this case, the trap stiffness $K_{i}$ (i.e., the eigen values of the force constant matrix $\mathbb{K}$ for $i=1,2$ or 3 ) can be complex numbers [42]. When $\operatorname{Re}\left[K_{i}\right]<0$, the trap is single potential well in the focal point. However, if $\operatorname{Im}\left[K_{i}\right] \neq 0$, the trap is unstable unless the environmental damping is larger than a critical value $\gamma_{\mathrm{c}}$. Figure 2(c) shows a typical stability diagram of the transverse motion of a core-shell particle trapped in a single $\mathrm{LG}_{03}$ beam. For the applications of optical tweezers in high vacuum, we are interested in the absolutely stable re- 

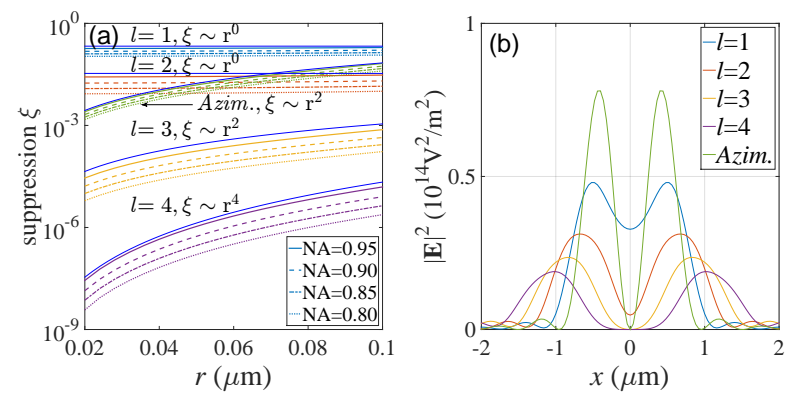

FIG. 3. (a) Light absorption suppression ratio $\xi$ for a core-shell sphere at the center of azimuthally and linearly polarized beams ( $\mathrm{LG}_{0 l}$ beams) relative to Gaussian beams, with different core radius $r$ and numerical aperture NA. Blue solid lines are for our approximate analytical results. $R=900 \mathrm{~nm}$, refractive index $n_{\text {diamond }}=$ $2.418+0.001 i$ and $n_{\text {sillica }}=1.458$ (absorption eliminated in this figure). (b) Field $|\mathbf{E}|^{2}$ distribution of incident strongly focused azimuthally and linearly polarized beams $\left(\mathrm{LG}_{0 l}\right.$ beams polarized along $x$ axis). All beams are with power $P_{1}=P_{2}=50 \mathrm{~mW}$ focused by the lens of NA $=0.95$.

gions (ASRs), where the particle can be trapped in the absence of any damping $(\gamma=0)$. In the case of the linearly polarized $\mathrm{LG}_{03}$ beam, there are several ASRs (e.g., around $R=1.5 \mu \mathrm{m}$ ),

The trapping stability depends on the beam polarization. Similar calculations are made for the azimuthally and circularly polarized beams [see Figs. 2(b) and 2(d)]. Being different from the linearly polarized beams, no ASR appears in the circularly polarized case. A finite damping rate $\gamma$ due to the environment (e.g., the collisions with the residual molecules) is necessary to maintain a stable trapping. In contrast, the azimuthally polarized beam provides a stable trap as long as the particle size exceeds the critical radius. This can be understood because the azimuthally polarized beam indeed does not carry OAM.

Counter-propagating beams with the same OAM improve the trapping stability. When the particle is displaced along $x$ direction, the $x$ components radiation force of the two beams add up, while the $y$ components are canceled. Figure 2(e) shows the stability diagram for two counter-propagating $\mathrm{LG}_{03}$ beams, with powers $P_{1}$ and $P_{2}=q P_{1}$ (with the power mismatch ratio $q$ ). The ASR is enlarged when the ratio $q$ approaches to unity. For example, particles with radius $R$ around $0.9 \mu \mathrm{m}$ and $R>1.3 \mu \mathrm{m}$ can be stably trapped even when the beam intensities are not perfectly matched (e.g., in the region with $0.9<q \leq 1$ ). In the circularly polarized case, the counter-propagating beams reduce the critical damping coefficient. Unfortunately, the ASR appears only when the beam intensities are exactly matched (i.e., $P_{1}=P_{2}$ or $q=1$ ). Accordingly, in terms of trapping stability, we conclude that the azimuthally polarized Gaussian beam and the linearly polarized LG beams are appropriate for the dual-beam optical tweezers in high vacuum. The cases for doughnut beams with different OAM and polarization have been investigated systematically and more details can be seen in supplementary information.

Heat absorption-. Having discussed the trapping stability, now we turn to the suppression of the heat absorption. Fig-
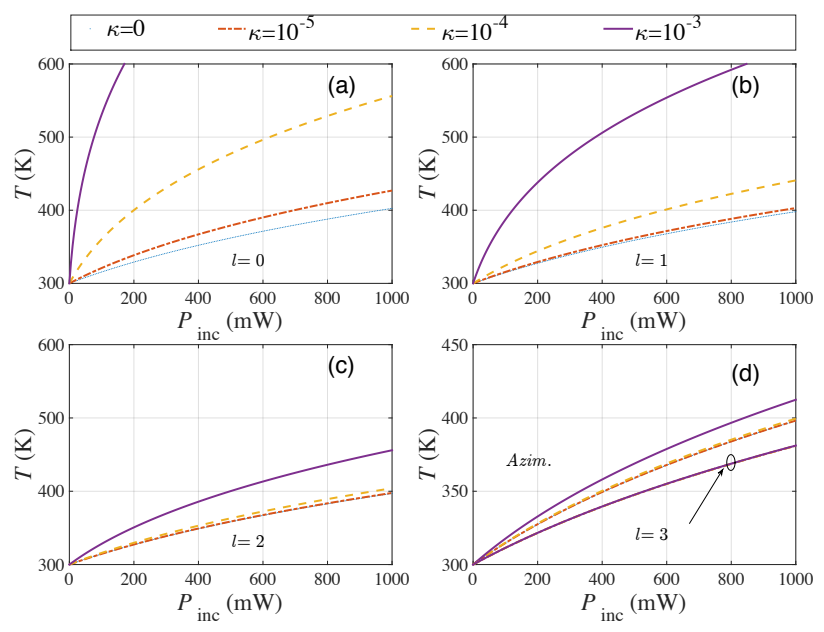

FIG. 4. The equilibrium temperature $T$ versus the incident laser power $P_{i n c}$ for a core-shell sphere trapped in linearly polarized $\mathrm{LG}_{0 l}$ beams and azimuthally polarized beam. Cases with (a) $l=0$, (b) $l=1$, (c) $l=2$, (d) $l=3$ and (d) azimuthally polarized beam are shown.

ure 3(a) shows the absorption coefficients $c_{\mathrm{abs}}$ of the a composite particle in azimuthally polarized Gaussian beam and linearly polarized $\mathrm{LG}_{0 l}$ beam with different OAM index $l$. All the absorption coefficients are normalized by the absorption coefficient $c_{\mathrm{abs}, 0}$ of the same particle in a Gaussian beam, and are thus defined as suppression ratio

$$
\xi \equiv \frac{c_{\mathrm{abs}}}{c_{\mathrm{abs}, 0}} .
$$

The heat absorption depends on both polarization and OAM. In the azimuthally polarized beam, the heat absorption of the particle is reduced by a factor of $10^{2}$ to $10^{3}$, depending on the size of the absorptive core. While, for the LG beams, the suppression ratio $\xi$ has qualitatively different behavior for different OAMs. In the cases of $l=1$ and $l=2$, the suppression effect is relatively weak and the ratio $\xi$ is independent on the radius of the core. However, for $l \geq 3$, the heat absorption is significantly reduced $\left(\xi<10^{-3}\right.$ for core radius $\left.r<100 \mathrm{~nm}\right)$, and follows a power-law dependence of the core size.

Different behavior of the heat suppression ratio originates from the intensity distribution of the incident strongly focused beams. Figure 3(b) shows the intensity distribution along the $x$ axis of the azimuthally polarized beam and LG beams with different OAMs. For the typical core radius $r<100 \mathrm{~nm}$, we expand the intensity distribution into power series of $k x$ (in the $x$ direction for example, and $k x \ll 1$ with the wave number $k=2 \pi / \lambda$ ) around the equilibrium position (i.e., the beam center). We find that, for the azimuthally polarized beam, the intensity exactly vanishes at the beam center $(x=0)$ and increases quadratically as increasing $x$, i.e., $I_{\mathrm{a}} \approx A_{\mathrm{a}}(k x)^{2}$. For the strongly focused $\mathrm{LG}_{0 l}$ modes, the intensity reads

$$
I_{l}(x) \approx A_{l}(k x)^{n_{l}}, \quad \text { for integer } l \geq 1 .
$$

Here, $A_{\mathrm{a}}$ and $A_{l}$ are the expansion coefficients. Being different from the paraxial $L G$ beams, the $\mathrm{LG}_{01}$ and the $\mathrm{LG}_{02}$ 
modes have finite intensity at the focal point (i.e., with constant leading terms with $n_{1}=n_{2}=0$ ). For the $\mathrm{LG}_{0 l}$ modes with $l \geq 3$, it is absolutely dark at the beam center $\left[I_{l \geq 3}(0)=0\right]$ and the expansion power index depends on the OAM index $l$ as $n_{l}=2(l-2)$. This explains the power-law behavior of the heat suppression ratio in Fig. 3(a), and suggests that the azimuthally polarized beam and the $\mathrm{LG}_{0 l}$ modes with $l \geq 3$ are good candidates for solving the thermal damage problem. Details of the intensity expansion and suppression approximations can be seen in supplementary information. Systematically investigation for right circularly polarized beams has also been included.

With the absorption coefficient $c_{\text {abs }}$, we estimate the equilibrium temperature $T$ of the composite particle trapped by different beams. We consider the vacuum environment of temperature $T_{0}$, where radiation is the dominating heat transfer mechanism. The absorbed energy from the beams is balanced by the black-body radiation as

$$
c_{\mathrm{abs}} P_{\mathrm{inc}}+\sigma A T_{0}^{4}=\sigma A T^{4},
$$

where $P_{\text {inc }}$ is the total incident power of the counterpropagating trapping beams, $\sigma$ is Stefan's constant and $A=$ $4 \pi R^{2}$ is the surface area of the sphere. Here, we have neglected the heat dissipation due to the surface conduction $j=k_{\mathrm{s}} A\left(T-T_{0}\right)$, where $k_{\mathrm{s}}$ is the surface conduct coefficient due to the residual gas. Accordingly, Eq. (3) gives an estimation of the upper bound of the equilibrium temperature. Figure 4 shows the equilibrium temperature $T$ of a stably trapped particle (denoted as a ' $X$ ' in Fig. $2(\mathrm{e}), R=900 \mathrm{~nm}$ ) as the function of incident power $P_{\text {inc }}$. We assume that the shell of the composite particle is made of silica with absorption coefficient $100 \mathrm{~dB} / \mathrm{km}$, corresponding to the imaginary part of the refractive index $\kappa_{\text {shell }}=2 \times 10^{-9}$. The diamond core could be very absorptive due to the intrinsic defects and the imperfect surface. We consider the imaginary part of refractive index of the diamond core ranging from $\kappa_{\text {core }} \sim 10^{-3}$ to $10^{-5}$. For the fundamental Gaussian mode (i.e., $l=0$ ), the temperature increases dramatically as increasing the incident power. However, when trapped by the doughnut beams, particularly the azimuthally polarized beam and the $\mathrm{LG}_{03}$ mode [see Fig. 4(d)], the diamond core has negligible contribution to the temperature increasement. The composite particle can afford much stronger power (up to the order of Watt) of the trapping beams, without significant heating effect.

In this part, we discuss two figures of merit of the system, namely, the trapping frequency $\Omega=\sqrt{K / M}$ and mechanical quality factor $Q=\Omega / \Gamma$ of the mechanical oscillation. Here $K$ is the force constant, $M$ is the mass of oscillator, and $\Gamma=\gamma / M$ is the damping coefficient with Stokes friction coefficient $\gamma$ due to the residual gas. Figures 5 presents the frequency $\Omega$ and the quality factor $Q$ for the azimuthally polarized beam and the linearly polarized $\mathrm{LG}_{03}$ beam, respectively. For a given particle size, the azimuthally polarized beam provides a trapping frequency in the order of $100 \mathrm{kHz}$, much higher than that of the $\mathrm{LG}_{03}$ mode. While the later creates an optical trap with non-degenerate frequencies, in the order $10 \mathrm{kHz}$, in the $x$ and $y$ directions. The quality factor of the mechanical oscillation $Q$ is inversely proportional to the damping coefficient $\gamma$ due to
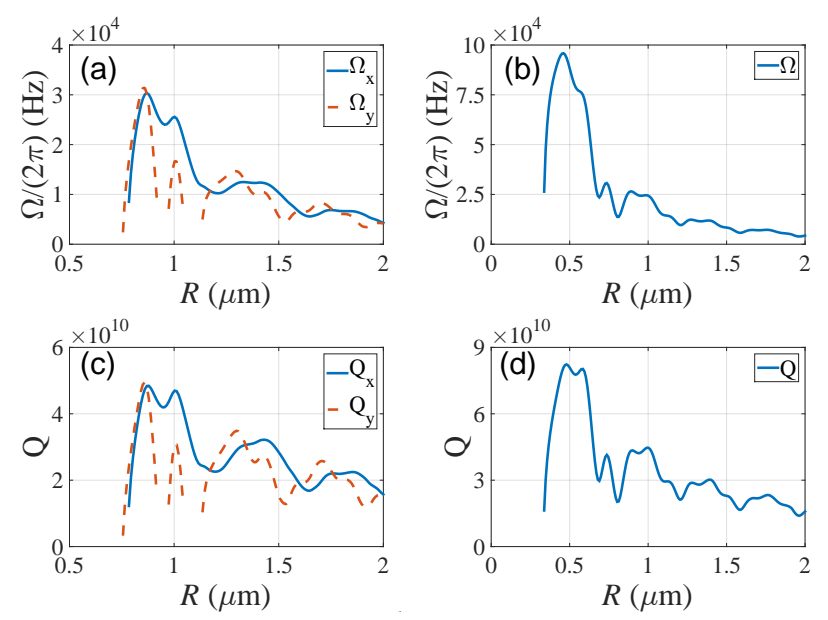

FIG. 5. The oscillation frequency $\Omega /(2 \pi)$ of the sphere in (a) linearly polarized $\mathrm{LG}_{03}$ beams and (b) azimuthally polarized beams. And the mechanical quality factor $Q$ of the sphere in (c) linearly polarized $\mathrm{LG}_{03}$ beams and (d) azimuthally polarized beams under pressure $10^{-6} \mathrm{~Pa}$. All beams are with total power $100 \mathrm{~mW}$ and $\mathrm{NA}=0.95$.

the residual gas. The quality factor reaches $Q \sim 10^{10}$, with a residual pressure $\mathcal{P}=10^{-6} \mathrm{~Pa}$. Reducing the pressure can further increase the quality factor, to the best figure of merit of this opto-mechanical system.

Conclusion-. Optical tweezers achieved great success in the past years in broad research fields ranging from biology, statistical physics, to microchemistry. However, the heat absorption problem prevents the exciting applications in the lowdissipation vacuum environment. We propose to use doughnut beams and core-shell particles to implement stable optical tweezers system in high vacuum. The low-absorptive shell (e.g., the silica shell) plays a role of 'sample-holder', which interacts with the trapping beam and provides the radiation force for levitation. While the 'sample' could be more general particles (not necessarily to be nanodiamonds). Once loaded in the sample-holder, no matter how strong absorptive it is, the core particle has little chance to see the trapping beam. With a systematic study of the physical effect of the beam OAM and polarization on the trapping stability and heat absorption, we provide a comprehensive solution to the heat absorption problem and make optical tweezers a powerful tool in many disciplines, especially in optomechanical applications and in the future quantum technologies.

\section{Appendix A: investigation method: Debye Integral and Lorentz-Mie Theory}

The investigation method is formulated concisely in this part. The strongly focused incident laser beam is modeled by the generalized vector Debye integral, and the generalized Lorentz-Mie theory (GLMT) is used to solve the scattering problem of light field. Finally, the time-averaged Maxwell stress tensor is used to calculate the force exerted on the sphere particle. 
The strongly focused beam is affected dramatically by the lens with high numerical aperture (NA), and cannot be described by the expression of paraxial beams. We model various doughnut beams, including linearly polarized LaguerreGaussian (lpLG) beams, right circularly polarized LaguerreGaussian (rcLG) beams and azimuthally polarized Gaussian (apG) beam here, by the highly accurate generalized vector Debye integral theory $[41,44]$. The incident field near the focus is formulated as:

$$
\begin{aligned}
\mathbf{E}(\rho, \varphi, z)= & -\frac{i k f e^{-i k f}}{2 \pi} \int_{0}^{\theta_{\max }} d \theta \sin \theta \int_{0}^{2 \pi} d \phi\left[\mathbf{E}_{\infty}(\theta, \phi)\right. \\
& \left.\times e^{i k \rho \sin \theta \cos (\phi-\varphi)+i k z \cos \theta}\right],
\end{aligned}
$$

where $\mathbf{E}_{\infty}(\theta, \phi)$ is the electric field vector on the Gaussian reference sphere, and the exponential factor is the phase accumulated during the light propagation. This expression has been used to investigate the properties of strongly focused beams and shows good accuracy [41, 42].

The scattering of incident beam is calculated by the GLMT $[44,45]$ and is formulated concisely here. In this theory, the incoming and outgoing fields are expanded in vector spherical wavefunctions (VSWFs):

$$
\begin{aligned}
\mathbf{E}^{\text {in }} & =\sum_{n=1}^{\infty} \sum_{m=-n}^{n}\left[a_{m n} \mathbf{M}_{m n}(k \mathbf{r})+b_{m n} \mathbf{N}_{m n}(k \mathbf{r}),\right. \\
\mathbf{E}^{\text {out }} & =\sum_{n=1}^{\infty} \sum_{m=-n}^{n}\left[p_{m n} \mathbf{M}_{m n}(k \mathbf{r})+q_{m n} \mathbf{N}_{m n}(k \mathbf{r}) .\right.
\end{aligned}
$$

The coefficients of incoming and outgoing fields are related by the T-matrix $\mathbb{T}$ of the particle as:

$$
\left(\begin{array}{l}
\mathbf{p} \\
\mathbf{q}
\end{array}\right)=\mathbb{T}\left(\begin{array}{l}
\mathbf{a} \\
\mathbf{b}
\end{array}\right) .
$$

For sphere or multi-layer sphere, $\mathbb{T}$ is diagonal and can be calculated directly or by iteration $[46,47]$.

The force and torque exerted on the sphere particle are calculated from time-averaged Maxwell stress tensor:

$$
\begin{aligned}
\overline{\mathcal{T}} & =\frac{1}{2} \operatorname{Re}\left[\varepsilon \mathbf{E}^{*} \mathbf{E}+\frac{\mathbf{B}^{*} \mathbf{B}}{\mu}-\frac{1}{2} \overleftrightarrow{1}\left(\varepsilon \mathbf{E}^{*} \cdot \mathbf{E}+\frac{\mathbf{B}^{*} \cdot \mathbf{B}}{\mu}\right)\right. \\
\mathbf{F} & =\oint d \mathbf{S} \cdot \overline{\mathcal{T}} \\
\mathbf{\Gamma} & =\oint d \mathbf{S} \cdot(\mathbf{r} \times \overline{\mathcal{T}})
\end{aligned}
$$

They are then formulated by the incident and scattered field coefficients, and finally we get, e.g,, the axial force and torque:

$$
\begin{aligned}
F_{z}= & \frac{n_{m} P}{c} \frac{2}{P_{c}} \sum_{n=1}^{\infty} \sum_{m=-n}^{n}\left\{\frac{m}{n(n+1)} \operatorname{Re}\left(a_{m n}^{*} b_{m n}-p_{m n}^{*} q_{m n}\right)\right. \\
& -\frac{1}{n+1}\left[\frac{n(n+2)(n-m+1)(n+m+1)}{(2 n+1)(2 n+3)}\right]^{1 / 2} \\
& \times \operatorname{Im}\left(a_{m n} a_{m n+1}^{*}+b_{m n} b_{m n+1}^{*}-p_{m n} p_{m n+1}^{*}\right. \\
& \left.\left.-q_{m n} q_{m n+1}^{*}\right)\right\}, \\
\tau_{z}= & \frac{P}{k c} \frac{2}{P_{c}} \sum_{n=1}^{\infty} \sum_{m=-n}^{n}[m \\
& \left.\times\left(a_{m n} a_{m n}^{*}+b_{m n} b_{m n}^{*}-p_{m n} p_{m n}^{*}-q_{m n} q_{m n}^{*}\right)\right] .
\end{aligned}
$$

Among the last two equations,

$$
P_{c}=\sum_{n=1}^{\infty} \sum_{m=-n}^{n}\left(a_{m n} a_{m n}^{*}+b_{m n} b_{m n}^{*}\right)
$$

is proportional to the incident power, $n_{m}$ is the refractive index of the medium surrounding the particle and $P$ is the power of the incident laser beam. Thus from the extinction and scattering coefficients, we get the absorption coefficient of the particle:

$$
\begin{aligned}
& c_{\mathrm{ext}}=-\frac{1}{P_{c}} \sum_{n=1}^{\infty} \sum_{m=-n}^{n} \operatorname{Re}\left(a_{m n} p_{m n}^{*}+b_{m n} q_{m n}^{*}\right), \\
& c_{\mathrm{sca}}=\frac{1}{P_{c}} \sum_{n=1}^{\infty} \sum_{m=-n}^{n}\left(p_{m n} p_{m n}^{*}+q_{m n} q_{m n}^{*}\right), \\
& c_{\mathrm{abs}}=c_{\mathrm{ext}}-c_{\mathrm{sca}} .
\end{aligned}
$$

\section{Appendix B: Phase diagram and Trapping stability in various doughnut beams}

Systematical investigation of the trapping stability in different doughnut beams has been made and is shown in this part. For a sphere in an optical trap, the force constant is a tensor $\mathbb{K}$ with elements [42]

$$
K_{i j}=\frac{\partial F_{i}}{\partial x_{j}}
$$

where $i, j=x, y, z$ are the Cartesian coordinates. The eigen vectors of $\mathbb{K}$ are the eigen-modes of sphere motion and can be used to analyze its stability. For a sphere in a LG beam, the force matrix can be written as

$$
\mathbb{K}=\left[\begin{array}{lll}
a & d & 0 \\
g & b & 0 \\
0 & 0 & c
\end{array}\right]
$$

The eigen motion modes along $z$ axis is independent of those on the transverse $x y$ plane. Using the theory of the trapping stability by optical vortex beam (i.e., LG beam) [42], various LG beams with different $l$ and polarizations have been investigated here.

Especially, the case of a single $\boldsymbol{x}$ polarized $\mathrm{LG}_{03}$ beam are shown in Fig. S1(a) for eigen force constant, in Fig. S1(b) for force constant elements and in Fig. 2(c) of the main text for phase diagram. It is noticed that, different from the phase diagram of $\mathrm{LG}_{01}$ and $\mathrm{LG}_{02}$ beam, there is a region which is always unstable despite of the environment damping at $R \sim$ $1.1 \mu \mathrm{m}$ [see Fig. 2(c)]. It can be seen from Fig. S1(b) that the force constant $b=K_{y y}$ is positive at $R \sim 1.1 \mu \mathrm{m}$.

The rcLG beams are different from lpLG beam as they have no stable window when $\gamma=0$, as shown in Fig. S2(b)(e)(h) for $l=1,2,3$, respectively. For different $q$, the minimum $\gamma_{c}$ needed to keep stable is shown in Fig. S2(c)(f)(i). 

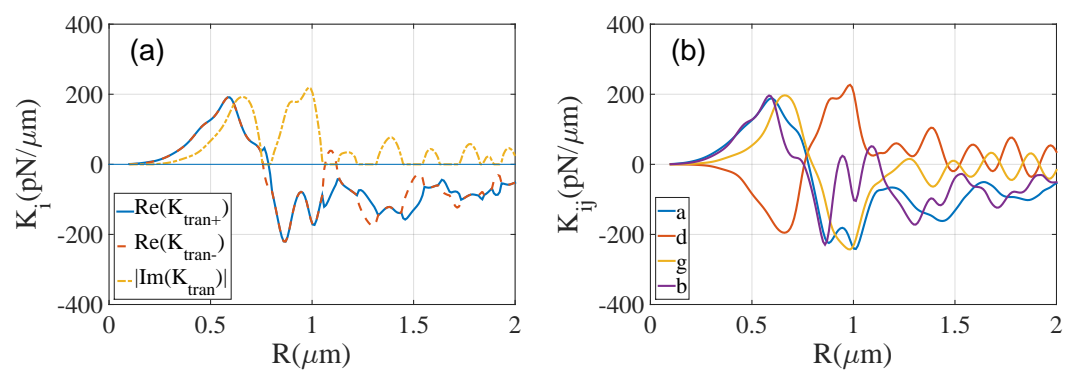

FIG. S1. (a) The transverse eigen force constant $K_{i}$ and (b) force constant element $K_{i j}$ for a core-shell sphere trapped in a single $\mathrm{LG}_{03}$ beam polarized along $\boldsymbol{x}$. All beams are with power $P=100 \mathrm{~mW}, \lambda=1064 \mathrm{~nm}$ and focused by lens with NA $=0.95$. All spheres are with core radius $r=20 \mathrm{~nm}$.
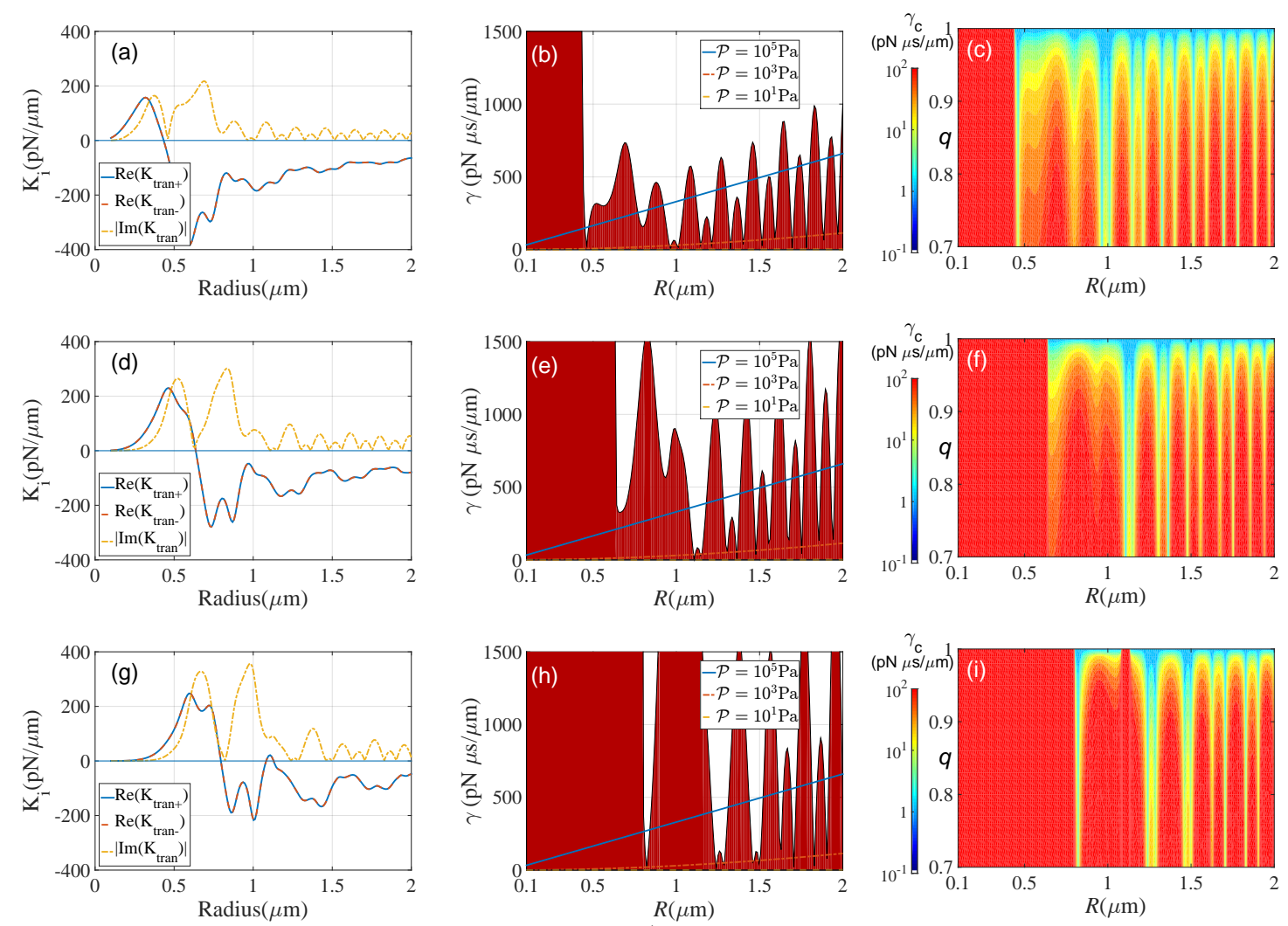

FIG. S2. (a) The transverse eigen force constant $K_{i}$ and (b) the phase diagram of a core-shell sphere in a single right circularly polarized $\mathrm{LG}_{01}$ beam. (c) Minimum friction $\gamma_{c}$ needed for stable trapping in two counter-propagating $\mathrm{rcLG}_{01}$ beams with different power ratio $q$. (d-f) The same as the first row, for $\mathrm{rcLG}_{02}$ beams. (g-i) The same as the first row, for $\mathrm{rcLG}_{03}$ beams. All beams are with power $P=100 \mathrm{~mW}$, $\lambda=1064 \mathrm{~nm}$ and focused by lens with NA $=0.95$. All spheres are with core radius $r=20 \mathrm{~nm}$.

\section{Appendix C: Approximate expression of the intensity near the focus}

The field of the strongly focused beam near the focus determines the absorption coefficients of the levitated core-shell sphere. Although there is no analytic expression of the field of the strongly focused beam, an approximate analytical expression near the beam focus will be helpful both for theory and experimental investigation. Our analytical approximations are shown in this part.
The field distribution of focused LG beam is expressed with vector Debye integral theory with Eq. (A1). When $z=0$, and

$$
\delta \triangleq k \rho \ll 1,
$$

we expand the field near the focus with Taylor series. For an $\hat{\boldsymbol{x}}$ direction linearly polarized incident $\mathrm{LG}_{0 l}$ beam, the light intensity $I_{l}=\frac{1}{2} \varepsilon_{m} \varepsilon_{0} v_{m}\left|\mathbf{E}_{l}\right|^{2}$ near the focus can be expressed as $I_{l}=A_{l} \delta^{n_{l}}, n_{l}=2(l-2)$. The absorption coefficients of a small sphere with radius $r$ can be approximated as 


$$
c_{\mathrm{abs}}=\int_{V} \eta_{l} \alpha_{l} I_{l} d V / P_{\mathrm{inc}},
$$

where $\alpha_{l}$ is the light attenuation coefficient in sphere, $I_{l}$ is the light intensity and $V$ is domain of the sphere core with radius $r . \eta_{l}$ is a correction factor considering the affection such as the changing of light field after scattering. The suppression coefficients ratio are defined as $\xi=c_{\mathrm{abs}} / c_{\mathrm{abs}, 0}$ and follow different power-law as $\xi_{l}=\xi_{l 0} \delta^{n_{l}}, n_{l}=2(l-2)$. Choosing $\eta_{0}=1.0, \eta_{1}=1.3, \eta_{2}=0.9, \eta_{3}=0.45$, and $\eta_{4}=0.40$ when $\mathrm{NA}=1.0$, we get $\xi_{10}=0.21, \xi_{20}=0.034, \xi_{30}=0.0032$, and $\xi_{40}=1.8 \times 10^{-4}$. The approximate analytical results of $\xi_{l}$ are plotted in Fig. 3(a) with blue lines and match the numerical results well.

Absorption suppression for rcLG beams shows similar behavior as those in lpLG beams. The absorption scale with $r$ can also be analyzed as before. The suppression coefficients follow different power-law as $\xi_{l}=\xi_{l 0} \delta^{n_{l}}, n_{l}=2 l$. The approximate results are also plotted in Fig. S3(a). Though the suppression is stronger in rcLG beams than that in $1 \mathrm{pLG}$ beams, the minimum $\gamma_{c}$ needed limits quality factor of the mechanical oscillation. So, the linear polarized LG beams are better for optomechanical application.

The apG beam also have zeros intensity in the beam focus. The phase diagram for a sphere in apG beam is shown in Fig. 2(b). Above the transition radius $R_{\text {tran }}=0.33 \mu \mathrm{m}$, the sphere is always stable. For apG beam, the suppression coefficient ratio can be written as $\xi_{a}=\xi_{a 0} \delta^{2}$. It is also plotted in Fig. 3(a).

\section{Appendix D: quality factor $Q$ in various doughnut beams and some discussions}

Doughnut beams have different field distributions ( especially, different radius of their bright rings) and thus have different trapping potential. The mechanical oscillation frequency $\Omega$ and quality factor $Q$ are investigated systematically. generally, larger radius of bright rings lead to smaller $\Omega$ and $Q$. The case for a spheres in linearly polarized $\mathrm{LG}_{0 l}$ beams with different $l$ is shown in Fig. S3.

\section{ACKNOWLEDGMENTS}

We are grateful to Prof. Zhifang Lin for sharing the numerical code. We thank Prof. Jack $\mathrm{Ng}$ for the helpful discussion. This work was supported by the NSFC grants (No. 11374032). J.C. is also supported by Shanxi Science and Technology Department through 2014011008-1, 2014021004 and NSFC through 11404201, 11674204.
[1] D. G. Grier, Nature 424, 810 (2003).

[2] A. Ashkin, J. M. Dziedzic, J. E. Bjorkholm, and S. Chu, Opt. Lett. 11, 288 (1986).

[3] A. Ashkin, J. M. Dziedzic, and T. Yamane, Nature 330, 769 (1987).

[4] A. Ashkin and J. Dziedzic, Science 235, 1517 (1987).

[5] K. Dholakia and P. Zemánek, Rev. Mod. Phys. 82, 1767 (2010).

[6] K. Dholakia, P. Reece, and M. Gu, Chem. Soc. Rev. 37, 42 (2008).

[7] D. E. Chang, C. a. Regal, S. B. Papp, D. J. Wilson, J. Ye, O. Painter, H. J. Kimble, and P. Zoller, Proc. Natl. Acad. Sci. 107, 1005 (2010).

[8] O. Romero-Isart, A. C. Pflanzer, F. Blaser, R. Kaltenbaek, N. Kiesel, M. Aspelmeyer, and J. I. Cirac, Phys. Rev. Lett. 107, 020405 (2011).

[9] T. Li, S. Kheifets, D. Medellin, and M. G. Raizen, Science 328, 1673 (2010).

[10] T. Li, S. Kheifets, and M. G. Raizen, Nat. Phys. 7, 527 (2011).

[11] S. Kheifets, A. Simha, K. Melin, T. Li, and M. G. Raizen, Science 343, 1493 (2014).

[12] J. Gieseler, B. Deutsch, R. Quidant, and L. Novotny, Phys. Rev. Lett. 109, 103603 (2012).

[13] J. Gieseler, R. Quidant, C. Dellago, and L. Novotny, Nat. Nanotechnol. 9, 358 (2014).

[14] J. Millen, T. Deesuwan, P. Barker, and J. Anders, Nat. Nanotechnol. 9, 425 (2014).

[15] V. Jain, J. Gieseler, C. Moritz, C. Dellago, R. Quidant, and L. Novotny, Phys. Rev. Lett. 116, 243601 (2016).

[16] Z.-q. Yin, A. A. Geraci, and T. Li, Int. J. Mod. Phys. B 27, 1330018 (2013).
[17] P. Rabl, P. Cappellaro, M. V. G. Dutt, L. Jiang, J. R. Maze, and M. D. Lukin, Phys. Rev. B 79, 041302 (2009).

[18] A. A. Geraci, S. B. Papp, and J. Kitching, Phys. Rev. Lett. 105, 101101 (2010).

[19] A. Arvanitaki and A. A. Geraci, Phys. Rev. Lett. 110, 071105 (2013).

[20] M. Scala, M. S. Kim, G. W. Morley, P. F. Barker, and S. Bose, Phys. Rev. Lett. 111, 180403 (2013).

[21] N. Zhao and Z.-q. Yin, Phys. Rev. A 90, 042118 (2014).

[22] Z.-q. Yin, T. Li, X. Zhang, and L. M. Duan, Phys. Rev. A 88, 033614 (2013).

[23] Z. Yin, N. Zhao, and T. Li, Sci. China-Phys. Mech. Astron. 58, 050303 (2015).

[24] L. P. Neukirch, E. V. Haartman, J. M. Rosenholm, and A. N. Vamivakas, Nat. Photon. 9, 653 (2015).

[25] T. M. Hoang, J. Ahn, J. Bang, and T. Li, Nat. Commun. 7, 12250 (2016).

[26] A. C. Frangeskou, A. T. M. A. Rahman, L. Gines, S. Mandal, O. A. Williams, P. F. Barker, and G. W. Morley, arXiv:1608.04724.

[27] H. S. Knowles, D. M. Kara, and M. Atatüre, Nat. Mater. 13, 21 (2014).

[28] J. Gieseler, L. Novotny, and R. Quidant, Nat. Phys. 9, 806 (2013).

[29] V. R. Horowitz, B. J. Aleman, D. J. Christle, A. N. Cleland, and D. D. Awschalom, Proc. Natl. Acad. Sci. 109, 13493 (2012).

[30] M. Geiselmann, M. L. Juan, J. Renger, J. M. Say, L. J. Brown, F. J. G. de Abajo, F. Koppens, and R. Quidant, Nat. Nanotechnol. 8, 175 (2013).

[31] L. P. Neukirch, J. Gieseler, R. Quidant, L. Novotny, and A. Nick Vamivakas, Opt. Lett. 38, 2976 (2013). 

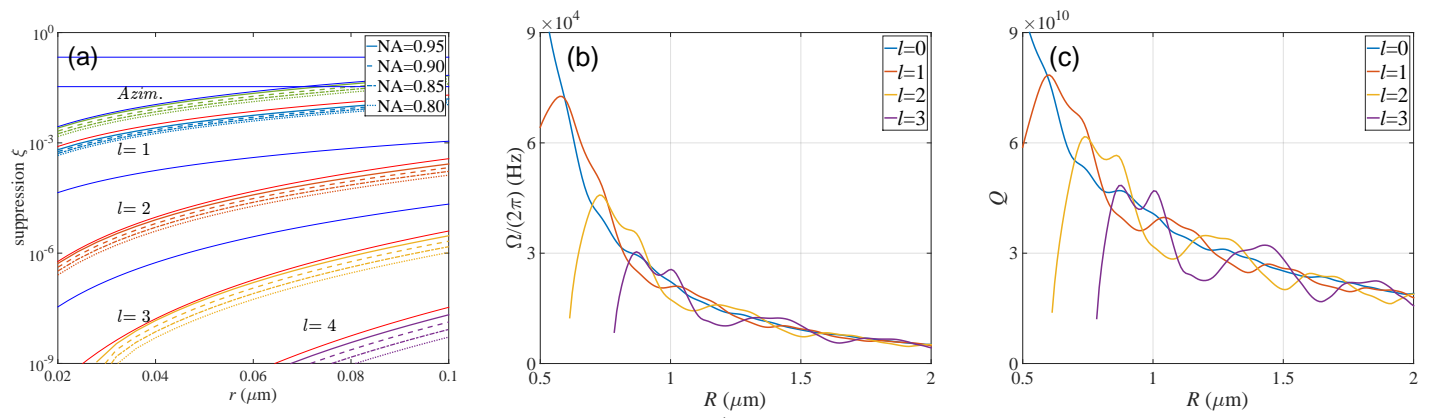

FIG. S3. (a) Light absorption suppression ratio $\xi$ for a core-shell sphere at the center of azimuthally and right circularly polarized beams ( $\mathrm{LG}_{0 l}$ beams) relative to linearly polarized Gaussian beams, with different core radius $r$ and numerical aperture NA. Red solid lines are for our analytical results. Blue lines are for linearly polarized LG beams. Other parameters are the same as Fig. 3(a). (b) The optomechanical frequency $\Omega /(2 \pi)$ and (c) mechanical quality factor $Q$ of a sphere in different lpLG beams along $x$ with power $100 \mathrm{~mW}$ under pressure $10^{-6} \mathrm{~Pa}$.

[32] A. T. M. A. Rahman, A. C. Frangeskou, M. S. Kim, S. Bose, G. W. Morley, and P. F. Barker, Sci. Rep. 6, 21633 (2016).

[33] E. von Haartman, H. Jiang, A. A. Khomich, J. Zhang, S. A. Burikov, T. A. Dolenko, J. Ruokolainen, H. Gu, O. A. Shenderova, I. I. Vlasov, and J. M. Rosenholm, J. Mater. Chem. B 1, 2358 (2013).

[34] H. He, N. Heckenberg, and H. Rubinsztein-Dunlop, J. Mod. Opt. 42, 217 (1995).

[35] K. T. Gahagan and G. a. Swartzlander, Opt. Lett. 21, 827 (1996).

[36] N. R. Heckenberg, M. E. J. Friese, T. A. Nieminen, and H. Rubinszteindunlop, in Optical Vortices, edited by M. Vasnetsov and K. Staliunas (Nova Science Publishers, 1999) pp. 75-105.

[37] D. Ganic, X. Gan, and M. Gu, Opt. Express 13, 1260 (2005).

[38] T. Otsu, T. Ando, Y. Takiguchi, Y. Ohtake, H. Toyoda, and H. Itoh, Opt. Rev. 22, 170 (2015).
[39] Y. Cao, T. Zhu, H. Lv, and W. Ding, Opt. Express 24, 3377 (2016).

[40] P. Jones, O. Maragó, and G. Volpe, Optical tweezers: Principles and applications (Cambridge University Press, 2015).

[41] L. Novotny and B. Hecht, Principles of nano-optics (Cambridge university press, 2012).

[42] J. Ng, Z. Lin, and C. T. Chan, Phys. Rev. Lett. 104, 103601 (2010).

[43] Noted that this is the phenomenological potential which is defined without distinguishing the gradient and scattering force.

[44] J. Chen, J. Ng, S. Liu, and Z. Lin, Phys. Rev. E 80, 026607 (2009).

[45] T. A. Nieminen, V. L. Y. Loke, A. B. Stilgoe, G. Knoner, A. M. Braczyk, N. R. Heckenberg, and H. Rubinsztein-Dunlop, J. Opt. A: Pure Appl. Opt. 9, S196 (2007).

[46] Z. S. Wu and Y. P. Wang, Radio Sci. 26, 1393 (1991).

[47] Z. S. Wu, L. X. Guo, K. F. Ren, G. Gouesbet, and G. Gréhan, Appl. Opt. 36, 5188 (1997). 